https://doi.org/10.35336/VA-2020-3-58-67

\author{
МЕСТО ХОЛТЕРОВСКОГО МОНИТОРИРОВАНИЯ ЭКГ В НОВЫХ РОССИЙСКИХ \\ РЕКОМЕНДАЦИЯХ ПО НАРУШЕНИЯМ РИТМА И ПРОВОДИМОСТИ СЕРДЦА \\ Ю.В.Шубик, М.М.Медведев, М.А.Батурова, А.Е.Ривин \\ Научно-клинический и образовательный центр «Кардиология» Санкт-Петербургского государственного \\ университета, Россия
}

Рассматриваются показания к проведению холтеровского мониторирования, которые вошли в новые российские рекомендации по нарушениям ритма и проводимости сердия, обсуждаются возможности методики в диагностике нарушений ритма и проводимости, определении лечебной тактики, контроле эффективности и безопасности лечения.

Ключевые слова: холтеровское мониторирование; электрокардиограмма; фибрилляция предсердий; трепетание предсердий; наджелудочковые тахикардии; желудочковые тахикардии; внезапная сердечная смерть; брадиаритмии

Конфликт интересов: авторы заявляют об отсутствии потенциального конфликта интересов Рукопись получена: 10.09.2020 Исправленная версия получена: 28.09.2020 Принята к публикации: 29.09.2020 Ответственный за переписку: Юрий Викторович Шубик, E-mail: yshubik@mail.ru

Для цитирования: Шубик ЮВ, Медведев ММ, Батурова МА, Ривин АЕ. Место холтеровского мониторирования ЭКГ в новых российских рекомендациях по нарушениям ритма и проводимости сердца. Вестник Аритмологии. 2020;27(3): 58-67. https://doi.org/10.35336/VA-2020-3-58-67.

\title{
ROLE OF HOLTER MONITORING IN THE NEW RUSSIAN GUIDELINES FOR ARRHYTHMIAS AND CONDUCTION DISTURBANCES
}

Yu.V.Shubik, M.M.Medvedev, M.A.Baturova, A.E.Rivin Medical Research and Educational Center "Cardiology"

The indications for Holter monitoring, which are included in the new Russian guidelines on cardiac arrhythmias and conduction disturbances, are considered. Possibilities of the diagnostic approaches of arrhythmias, management algorithms and treatment efficacy and safety monitoring are discussed.

Key words: Holter monitoring; electrocardiography; atrial fibrillation; atrial flutter; supraventricular tachycardia; ventricular tachycardia; sudden cardiac death; bradyarrhythmia

Conflict of interests: nothing to declare

Received: 10.09.2020 Corrected version received: 28.09.2020 Accepted: 29.09.2020

Corresponding author: Yuri Shubik, E-mail: yshubik@mail.ru

For citation: Shubik YuV, Medvedev MM, Baturova MA, Rivin AE. Role of Holter monitoring in the new Russian guidelines for arrhythmias and conduction disturbances. Journal of Arrhythmology. 2020;27(3): 58-67. https://doi. org/10.35336/VA-2020-3-58-67.

В конце 2020 - начале 2021 года появятся новые рекомендации по диагностике и лечению нарушений ритма и проводимости сердца. В настоящее время практически завершена подготовка следующих документов:

• «Фибрилляция и трепетание предсердий»;

- «Наджелудочковые тахикардии»;

- «Желудочковые нарушения ритма. Желудочковые тахикардии и внезапная сердечная смерть»;

- «Брадиаритмии и нарушения проводимости».

Очевидно, что эти рекомендации самым существенным образом отличаются от тех, с которыми мы были знакомы ранее. В основе каждого из документов лежат привычные нам т.н. «тезис-рекомендации». В некоторых случаях они сопровождаются комментария- ми. Главное отличие новых рекомендаций заключается в том, что все они станут официальным документом Министерства Здравоохранения РФ, обязательным к исполнению. Над каждым из них работала группа авторитетных специалистов, однако впервые «правила игры» определялись ФГБУ «Центр экспертизы и контроля качества медицинской помощи» (ЦЭККМП) Минздрава России. В частности, этим учреждением были предложены разработанные им новые классы рекомендаций и доказательные уровни, значительно отличающиеся от привычных нам, принятых Европейским обществом кардиологов (ЕОК). Поэтому, прежде чем переходить к обсуждению содержательной части, тезис-рекомендациям, касающимся холтеровского мониторирования (XМ) электрокардиограммы (ЭКГ), 
необходимо изложить принципы определения уровней достоверности доказательств (УДД) и уровней убедительности рекомендаций (УУР), предложенные ЦЭККМП не только для кардиологии, но и для других медицинских специальностей $[1,2]$.

Итак, УДД - степень уверенности в том, что найденный эффект от применения медицинского вмешательства является истинным. Приведем шкалу определения УДД для диагностических вмешательств (табл. 1).

УУР - степень уверенности в достоверности эффекта вмешательства и в том, что следование рекомендациям принесет больше пользы, чем вреда в конкретной ситуации. Ниже приведена шкала определения УУР для диагностических вмешательств (табл. 2).

Примем к сведению эти новые принципы формирования классов рекомендаций и доказательных уровней без пространного обсуждения: это не является целью настоящей публикации. Отметим только, что рабочие группы, ответственные за каждый из обсуждаемых документов, столкнулись с большими трудностями при попытке удовлетворить требования ЦЭККМП. В качестве единственного примера, имеющего непосредственное отношение к диагностике аритмий, приведем следующий. Казалось бы, нет никаких сомнений в необходимости регистрации ЭКГ при восстановлении синусового ритма у пациентов с пароксизмом фибрилляции предсердий (ФП) или желудочковой тахикардии? Однако придется согласиться с тем, что обнаружить на эту тему какие-либо систематические обзоры исследований, да еще с контролем референсным методом, представляется весьма трудной задачей. А раз так, не стоит удивляться, что в целом ряде случаев УУР и УдД могут оказаться весьма низкими, в т.ч. - самыми низкими. Впрочем, каждая из тезис-рекомендаций будет завершаться не только «российскими» показателями, но и - для сведения - привычными для нас классами рекомендаций и доказательными уровнями ЕОК (при наличии таковых).
Рассмотрим последовательно значение ХМ ЭКГ в диагностике и лечении нарушений ритма и проводимости сердца, учитывая при этом, что обсуждаем близкие к завершению, но все же не окончательные варианты рекомендаций.

\section{ФИБРИЛЛЯЦИЯ И ТРЕПЕТАНИЕ ПРЕДСЕРДИЙ}

В разделе «Диагностика», посвященном жалобам и анамнезу, представлены следующие тезис-рекомендации, имеющие отношение к ХМ ЭКГ.

1. При наличии жалоб на эпизоды неритмичного сердцебиения и при отсутствии документированных эпизодов ФП или трепетания предсердий (ТII) при записи ЭКГ рекомендуется выполнение ХМ ЭКГ для исключения коротких пароксизмов ФП/ТП (УУР С, УДД 5, ЕОК нет).

В комментарии указано, что при наличии эпизода наджелудочковой тахиаритмии длительностью более 30 секунд с характерными ЭКГ признаками ФП/ТП, пациенту должен быть установлен соответствующий диагноз и приняты все надлежащие меры по профилактике повторных пароксизмов и тромбоэмболических осложнений.

Из этой рекомендации, видимо, следует сделать два принципиально важных вывода. Первый заключается в том, что диагноз ФП и/или ТП не может быть установлен на основании жалоб пациента, какой бы яркой ни была клиническая картина: необходимо документальное подтверждение, без которого, в частности пациенту не могут быть назначены пероральные антикоагулянты. Такая рекомендация будет считаться ошибочной и может повлечь за собой (особенно в случае осложнений антикоагулянтной терапии) самые неприятные последствия. Второй вывод: выявление при ХМ ЭКГ пароксизмов наджелудочковой тахиаритмии продолжительностью $\leq 30$ секунд, даже если по результатам 12-канальной записи не остается никаких сомнений в характере аритмии, не позволяет ее иденти-

Таблица 1.

\section{Шкала оценки УДД для диагностических вмешательств}

\begin{tabular}{|l|l|}
\hline УдД & Иерархия дизайнов клинических исследований по убыванию уровня достоверности доказательств от 1 до 5 \\
\hline 1 & Систематические обзоры исследований с контролем референсным методом \\
\hline 2 & Отдельные исследования с контролем референсным методом \\
\hline 3 & $\begin{array}{l}\text { Исследования без последовательного контроля референсным методом или исследования с референсным } \\
\text { методом, не являющимся независимым от исследуемого метода }\end{array}$ \\
\hline 4 & Несравнительные исследования, описание клинического случая \\
\hline 5 & Имеется лишь обоснование механизма действия или мнение экспертов \\
\hline
\end{tabular}

таблица 2.

\section{Шкала определения УУР для диагностических вмешательств}

\begin{tabular}{|l|l|}
\hline УУР & Расшифровка \\
\hline А & $\begin{array}{l}\text { Однозначная (сильная) рекомендация (все исследования имеют высокое или удовлетворительное } \\
\text { методологическое качество, их выводы по интересующим исходам являются согласованными) }\end{array}$ \\
\hline В & $\begin{array}{l}\text { Неоднозначная (условная) рекомендация (не все исследования имеют высокое или удовлетворительное } \\
\text { методологическое качество и/или их выводы по интересующим исходам не являются согласованными) }\end{array}$ \\
\hline С & $\begin{array}{l}\text { Низкая (слабая) рекомендация - отсутствие доказательств надлежащего качества (все исследования имеют } \\
\text { низкое методологическое качество и их выводы по интересующим исходам не являются согласованными) }\end{array}$ \\
\hline
\end{tabular}


фицировать как пароксизмальную ФП. Это положение, полностью, впрочем, соответствующее рекомендациям ЕОК 2016 г [3], существенно затрудняет диагностику ФП: ведь оно не позволяет установить правильный диагноз во многих случаях, например, у пациентов с недокументированными продолжительными приступами неритмичного сердцебиения в анамнезе. В таких случаях диагностический поиск должен быть продолжен с использованием многосуточного ХМ ЭКГ или носимого «регистратора событий». Это досадное недоразумение, затрудняющее практическую работу кардиологов и терапевтов, устранено в совсем недавно появившихся рекомендациях ЕОК 2020 г [4]. Из них следует, что «...диагностическим признаком клинически значимой ФП является отсутствие закономерного повторения зубцов Р и наличие нерегулярных интервалов RR (если AB проводимость не нарушена), зафиксированные при стандартной записи ЭКГ в 12-ти отведениях или регистрации ЭКГ в одном отведении продолжительностью $\geq 30$ секунд». Можно ожидать, что формулировка в российских рекомендациях будет приведена в соответствие с европейской либо в обсуждаемой версии, либо в следующей.

2. Выполнение длительного мониторирования ЭКГ рекомендовано у некоторых симптомных пациентов для оценки адекватности контроля частоты и уточнения взаимосвязи между приступами ФП и жалобами (УУР С, УДД 5, ЕОК ІІаС).

В данном случае, как можно видеть, речь идет о банальном сопоставлении жалоб пациента с электрокардиографической картиной, необходимость которого не вызывает сомнений. У пациентов с постоянной формой ФП, жалующихся на сердцебиение, может быть оценена необходимость в контроле частоты сердечных сокращений (ЧСС). Кроме того, ХМ ЭКГ позволяет с одной стороны определить причину субъективного ощущения сердцебиения, которое совершенно не обязательно связано с ФП, с другой - выявить ФП у бессимптомных пациентов.

3. У пациентов с транзиторной ищемической атакой или ишемическим инсультом рекомендуется скрининг с помощью регистрации кратковременной ЭКГ с последующим мониторированием ЭКГ в течение как минимум 72 часов для выявления ФП (УУР А, УДД 2, ЕОК ІВ).

Эта тезис-рекомендация предусматривает необходимость длительного ХМ ЭКГ всем без исключения больным, ишемический инсульт или транзиторная ишемическая атака которых носят криптогенный характер. Целесообразно, видимо, напомнить, что принято выделять следующие механизмы нарушений мозгового кровообращения по ишемическому типу:

- атеротромботический ишемический инсульт - по данным дуплексного сканирования сосудов или ангиографии определяется стеноз интракраниальных или экстракраниальных артерий более 50\%, а по данным обследования сердца отсутствуют источники кардиоэмболии;

- кардиоэмболический ишемический инсульт - при потенциальном наличии источников эмболии в сердце (ФП, клапанные пороки сердца, инфаркт миокарда в острой стадии, синдром слабости синусового узла, дилатационная кардиомиопатия, инфекционный эндокардит, миксома левого предсердия) при отсутствии стенозирующего атеросклеротического поражения интракраниальных и экстракраниальных сосудов;

- лакунарный ишемический инсульт - нарушение мозгового кровообращения, обусловленное поражением небольших перфорирующих артерий;

- криптогенный - ишемический инсульт неустановленной этиологии.

При этом криптогенным ишемический инсульт считается в двух случаях: если возможны и атеротромботическая, и кардиоэмболическая причины (например, стенозирующий атеросклеротический процесс в интра- или экстракраниальных артериях у пациента с $Ф П)$, или если не установлена ни одна из них. Именно последним необходимо многосуточное ХМ ЭКГ для подтверждения или исключения ФП. Продолжительность исследования в 72 часа определена как минимальная: часто его проводят более длительно, семь суток и более. Очевидно, что выявление ФП повлечет за собой радикальное изменение антитромботической терапии и потребует назначения пероральных антикоагулянтов, т.к. даже в отсутствие всех прочих факторов риска тромбоэмболий транзиторная ишемическая атака или ишемический инсульт - это сразу 2 балла по шкале $\mathrm{CHA}_{2} \mathrm{DS}_{2}$-VASc. Отметим также, что визуализирующие методы обследования не помогут в дифференциальной диагностике, т.к. при компьютерной или магнитнорезонансной томографии атеротромботический и кардиоэмболический ишемический инсульт выглядят одинаково.

В разделе «Лечение» ХМ ЭКГ упоминается в следующих тезис рекомендациях.

1. Индивидуальный выбор препарата и его суточной дозы для контроля частоты ритма желудочков при ФП должен быть ориентирован на достижение целевых значений ЧСС: при полном отсутствии симптомов аритмии частота желудочковых сокращений в состоянии покоя должна быть не выме 110 ударов в минуту; при наличии симптомов, связанных с аритмией, - не выше 80 ударов в минуту (УУР А, УДД 3, ЕОК ІІаВ).

В комментарии к этой тезис-рекомендации указано, что индивидуальный контроль эффективности и безопасности такого лечения с учетом риска развития клинически значимой брадикардии, особенно в ночные часы, следует проводить с использованием ХМ ЭКГ, что делает метод основным для контроля ЧСС. При этом указано, что у больных с высоким уровнем двигательной активности (преимущественно молодые пациенты) эффективность назначенной терапии должна оцениваться с использованием проб с физической нагрузкой на велоэргометре или тредмиле.

2. Пациентам, длительно принимающим антиаритмическую терапию (АAT), рекомендован регулярный контроль длительности интервалов $P Q, Q R S, Q T$ и ЧСС с помощью ЭКГ и ХМ ЭКГ для оценки возможного риска проаритмического действия антиаритмических препаратов (ААП) и своевременного выявления клинически значимых 
нарущений проводимости и/или дисфункции синусового узла, обусловленных действием ААП. В начале лечения (первые 3 дня) рекомендован ежедневный контроль ЭКГ для обоснования адекватности выбора индивидуальной дозы препарата. При использовании амиодарона такой контроль рекомендовано дополнительно проводить через 1 и 4 недели (УУР А, УДД 2, ЕОК IВ).

Тезис-рекомендация в данном случае подчеркивает приоритет безопасности при назначении ААП пациентам с пароксизмальной и персистирующей ФП. В комментарии к ней сказано, что длительная ААТ не рекомендуется пациентам с интервалом QT > 500 мс или синусовой брадикардией менее 50 уд/мин в покое, эпизодами синоатриальной блокады II-III ст. или атриовентрикулярной (AB) блокады II-III ст. без предварительной имплантации электрокардиостимулятора. Очевидно, что оптимальным методом контроля безопасности ААТ является ХМ ЭКГ.

3. Пациентам, для которых на основании их убеждений и предпочтений принято решение о прекрацении антикоагулянтной терапии, рекомендовано длительное или частое мониторирование ЭКГ для выявления возможных рецидивов ФП (УУР С, УДД 5, ЕОК IIbC).

Из комментария к рекомендации следует, что решения о продолжении системной антикоагулянтной терапии после катетерной аблации должны базироваться на стратификации риска инсульта, а не на эффективности проведенной процедуры. Пациентам это должно быть разъяснено! Эта тезис-рекомендация имеет непосредственное отношение к интервенционному лечению ФП. Как известно, в случае успеха процедуры у пациентов с высоким риском тромбоэмболий лечение антикоагулянтами все равно должно быть продолжено «неопределенно долго». Это связано с вероятностью у них бессимптомных пароксизмов ФП, а также поздних, спустя продолжительное время после катетерной аблации, рецидивов. Понятно, что пациент может настаивать на прекращении лечения антикоагулянтами. Но такое решение может быть принято только т.н. мультидисциплинарной командой, которая должна убедиться в отсутствии пароксизмов ФП по данным ХМ ЭКГ.

Еще одно упоминание о ХМ ЭКГ можно увидеть в разделе, посвященном ФП у спортсменов.

Рекомендовано оценивать частоту жсллуочковых сокращений во время тренировки у каждого спортсмена с ФП (на основании симптомов и/или по данным суточного мониторирования) с последующим достижением целевых значений (УУР С, УДД 5, ЕОК IIaC).

Естественно, ХМ ЭКГ является самым удобным методом контроля ЧСС у этой категории пациентов.

В тезис-рекомендациях раздела «Профилактика и диспансерное наблюдение» ХМ ЭКГ не упоминается, однако суточное или многосуточное мониторирование включено здесь в перечень обязательных методов первичного обследования при впервые выявленной ФП или при подозрении на нее (наряду с ЭКГ, эхокардиографией, клиническим и биохимическим анализами крови, определением уровня тиреотропного гормона и гормонов щитовидной железы). Кроме того, предложено проводить суточное или многосуточное ХМ ЭКГ с целью оценки эффективности ААТ, выявления связи симптомов с эпизодами аритмии или диагностики других значимых нарушений ритма и проводимости.

В разделе «Организация медицинской помощи» при обсуждении показаний для плановой госпитализации обсуждаются пациенты с формированием тахикардиомиопатии на фоне ФП. Они должны быть госпитализированы для подбора пульс-урежающей терапии. При этом наличие тахисистолии у них должно быть зарегистрировано на ЭКГ или в покое при ХМ ЭКГ.

Во всех рассматриваемых нами рекомендациях, безусловно, самым важным является небольшой раздел «Критерии оценки качества медицинской помощи». Он включает не менее десяти положений, соблюдение которых считается абсолютно обязательным при обследовании и лечении каждого конкретного пациента с той или иной патологией. Соответственно, несоблюдение этих положений будет считаться врачебной ошибкой. Нелишним, видимо, будет напомнить, что понятие «врачебная ошибка» трактуется медицинским сообществом и, например, Следственным комитетом РФ - скажем осторожно - неодинаково. В рекомендациях «Фибрилляция и трепетание предсердий» одно из положений, по которым будет оцениваться качество оказания медицинской помощи, сформулировано следующим образом: проведены электрокардиографические методы исследования (ЭКГ, ХМ ЭКГ).

\section{НАДЖЕЛУДОЧКОВЫЕ ТАХИКАРДИИ}

Показания к ХМ ЭКГ у пациентов с наджелудочковыми тахикардиями (НЖТ) и подозрением на их наличие в целом, как и следует ожидать, похожи на таковые при ФП, однако имеют свои особенности. Так, в разделе «Инструментальные диагностические исследования» план обследования пациентов с подозрением на нефизиологическую синусовую тахикардию, наряду с ЭКГ, тестом с физической нагрузкой (для документирования неадекватной реакции ЧСС на минимальные физические нагрузки), трансторакальной эхокардиографией (для исключения структурной патологии сердца), включает ХМ ЭКГ: для определения средней ЧСС, ее изменений в зависимости от активности, оценки соотношения ЧСС и симптомов. Здесь же указаны критерии диагностики синусовой тахикардии по данным ХМ ЭКГ, указывающие на ее непароксизмальный характер: постепенное увеличение и снижение ЧСС. Кроме того, упомянуто о коротких бессимптомных пароксизмах фокусной предсердной тахикардии, которая часто выявляется при ХМ ЭКГ и не требует лечения.

Естественно, ХМ ЭКГ, как суточное, так и многосуточное, предлагается использовать в числе других электрокардиографических методов исследования (ЭКГ, носимых и имплантируемых «регистраторов кардиологических событий» и т. д.) для диагностики НЖТ в целом.

Тезис-рекомендации в разделе «Инструментальные диагностические исследования» сформулированы следующим образом. 
1. В тех случаях, когда анамнез или физикальные данные позволяют предположить НЖТ, ее наличие рекомендуется подтвердить с помощью ЭКГ или какой-либо другой методики с регистрацией электрокардиосигнала - суточное или многосуточное ХМ ЭКГ, носимый или имплантируемый «регистратор событий» и др. (УУР В, УДД 2, ЕОК нет).

В комментарии специально указано, что не самые высокие уровень убедительности рекомендаций и уровень достоверности доказательств обусловлены настолько очевидной и необсуждаемой необходимостью подтверждения диагноза НЖТ с помощью ЭКГ или какой-либо другой методики регистрации электрокардиосигнала, что доказательная база в доступной литературе оказалась весьма ограниченной.

2. У больных, получающих АAT, рекомендуется регулярная регистрация ЭКГ в 12 отведениях и/ или холтеровское мониторирование ЭКГ для оценки ее эффективности и безопасности (УУР С, УДД 5, EОК нет).

Тезис-рекомендация отражает очевидную необходимость контроля эффективности и безопасности применения ААП.

В разделе «Профилактика и диспансерное наблюдение, медицинские показания и противопоказания к применению методов профилактики» обсуждается значение наджелудочковых экстрасистол как триггера НЖТ и ФП, в частности, характеристики «нормальной» и «чрезмерной» эктопической активности. В качестве критерия высокого «бремени» предложено их количество более 500 по данным ХМ ЭКГ. Отметим, что на страницах журнала «Вестник аритмологии» также обсуждается важный вопрос «нормы» и «патологии» при нарушениях ритма и проводимости сердца [5].

В этом же разделе предложено использовать суточное и многосуточное ХМ ЭКГ в рамках планового обследования пациентов с НЖТ не реже 1 раза в год.

Ниже представлены тезис-рекомендации раздела «Профилактика и диспансерное наблюдение, медицинские показания и противопоказания к применению методов профилактики», имеющие отношение к ХМ ЭКГ.

1. Комплексная модификация факторов сердечно-сосудистого риска (лечение артериальной гипертензии, снижение избыточной массы тела, выявление и коррекция синдрома апноэ сна) рекомендуется пациентам с высоким бременем наджелудочковой экстрасистолии для снижения риска возникновения НЖТ (УУР С, УДД 4, ЕОК ІІаС).

Эта тезис-рекомендация тоже имеет непосредственное отношение к высокой предсердной эктопической активности. Из комментария к ней следует, что вопрос о влиянии лечения пациентов с высоким бременем предсердной экстрасистолии с помощью ААП или катетерной аблации на риск развития НЖТ, инсульта и смертельного исхода является дискутабельным. Очевидно, тем не менее, что количество и характер наджелудочковых эктопий должны быть определены. Кроме того, в числе факторов сердечно-сосудистого риска, требующих коррекции, указан, как можно видеть, синдром апноэ сна. Нам хорошо известно, что для его вы- явления в качестве скринингового метода используется обычное ХМ ЭКГ, позволяющее оценить реопневмограмму. Для более точной диагностики существует т.н. кардиореспираторное мониторирование, включающее, помимо ЭКГ, регистрацию движения дыхательных мышц грудной и/или брюшной стенки, дыхательного потока, храпа, сатурации кислорода, двигательной активности и положения тела пациента.

2. Суточное (или многосуточное) амбулаторное мониторирование ЭКГ рекомендуется для диагностики вызванной тахикардией кардиомиопатии путем выявления субклинических или интермиттирующих аритмий (УУР А, УДД 4, ЕОК ІІа В).

В данном случае, так же, как и при ФП, ХМ ЭКГ необходимо для оценки «бремени» и ЧСС у пациентов с НЖТ. Высокий «удельный вес» НЖТ и/или высокая ЧСС могут привести к формированию тахикардиомиопатии, требующей лечения. Это касается в том числе асимптомных или малосимптомных пациентов.

3. Неинвазивная оценка проводящих свойств дополнительного АВ соединения рекомендуется $у$ лиц с бессимптомным предвозбуждением (УУР В, УДД 3, ЕОК ІІьВ).

В комментарии к этой тезис-рекомендации указано, в частности, что низкий риск развития быстрой проводимости возбуждения по дополнительному АВ соединению и угрожающих жизни желудочковых аритмий в ответ на индуцируемую $\mathrm{AB}$ реципрокной тахикардией ФП характерен для интермиттирующего проведения возбуждения по дополнительному АВ соединению. Определение низкого риска возможно с помощью таких неинвазивных исследований, как ХМ ЭКГ (собственно интермиттирующее проведение) и теста с физической нагрузкой (исчезновение признаков предвозбуждения желудочков). При этом интермиттирующее предвозбуждение желудочков признается несовершенным маркером низкого риска, т.к. более одной пятой таких пациентов имеют короткий $(<250$ мс) эффективный рефрактерный период дополнительного АВ соединения.

\section{ЖЕЛУДОЧКОВЫЕ НАРУШЕНИЯ РИТМА. ЖЕЛУДОЧКОВЫЕ ТАХИКАРДИИ И ВНЕЗАПНАЯ СЕРДЕЧНАЯ СМЕРТЬ}

Рекомендации по диагностике и лечению желудочковых аритмий изложены таким образом, что в разделе «Инструментальная диагностика» упоминание о ХМ ЭКГ практически отсутствует. Лишь в одной из тезис-рекомендаций сказано следующее.

При редких симптомах (к примеру, синкопальные состояния) и невозможности регистрации ЭКГ во время эпизода холтеровским мониторированием рекомендовано использование наружного или имплантируемого петлевого регистратора для долговременной регистрации электрической активности проводящей системы сердца с целью выявления возможных транзиторных аритмий, способных вызывать такие симптомы (УУР В, УДД 3, ЕОК ІА).

Из этого определения следует, что одним из основных методов диагностики желудочковых аритмий все же является ХМ ЭКГ. Кроме того, дополнительно 
при обсуждении наследственных первичных аритмий из описания катехоламинергической полиморфной желудочковой тахикардии следует, что для установления диагноза рекомендуется ХМ ЭКГ и/или тест с физической нагрузкой, во время которого появляются предсердные и желудочковые аритмии, в т.ч. двунаправленная или полиморфная желудочковая тахикардия.

Тем не менее, в самом важном разделе рекомендаций, «Критерии оценки качества медицинской помощи», одно из положений, обязательных к исполнению, сформулировано следующим образом: выполнено ЭКГ исследование покоя в 12 отведениях и/или ХМ ЭКГ пациентам при прохождении обследования на предмет выявления желудочковых аритмий.

Большинство упоминаний о ХМ ЭКГ содержит раздел рекомендаций «Лечение». Во всех случаях они касаются желудочковой экстрасистолии (ЖЭ).

1. У пациентов без структурной патологии сердца / дисфункции левого желудочка (ЛЖ) лекарственное лечение ЖЭ рекомендовано в случаях, когда аритмия сопровождается клинической симптоматикой либо приводит к дилатации полостей сердца и снижению сократимости миокарда ЛЖ на фоне частой желудочковой эктопической активности, превышающей $15 \%$ от общего количества сердечных сокращений в сутки по данным ХМ ЭКГ (УУР A, УДД 3, ЕОК IC).

В данном случае принципиально важным является критерий необходимости ААТ вне зависимости от симптомности ЖЭ: $15 \%$ от всех сердечных сокращений в сутки. Он примерно соответствует критерию, который предлагается в некоторых других рекомендациях: 20000 ЖЭ в сутки [6]. Отметим, что это же количество эктопических сокращений является показанием для катетерной аблации ЖЭ.

2. Лекарственная ААТ ЖЭ у пациентов со структурной патологией сердца / дисфункцией ЛЖ рекомендована в случаях, когда аритмия сопровождается клинической симптоматикой либо приводит к дилатации полостей сердца и снижению сократимости миокарда ЛЖ на фоне частой желудочковой эктопической активности, превышающей 15\% от общего количества сердечных сокращений в сутки по данным ХМ ЭКГ (УУР А, УДД 3, ЕОК ІС).

Эта тезис-рекомендация ничем не отличается от предыдущей, в которой речь идет о т.н. «идиопатической» ЖЭ. Но в данном случае обсуждается лечение ЖЭ у пациентов со структурной патологией сердца и/ или дисфункцией ЛЖ.

3. У пациентов с имплантированными устройствами для проведения сердечной ресинхронизирующей терапии применение лекарственной ААТ рекомендовано для подавления частой ЖЭ в случаях, когда она приводит к снижению процента навязанного бивентрикулярного ритма ниже рекомендованного уровня (УУР В, УДД 2, ЕОК ІІаВ).

В комментарии к данной тезис-рекомендации сказано, что для достижения наилучшего эффекта сердечной ресинхронизирующей терапии необходимо стремиться к максимально высокому проценту навязанного бивентрикулярного ритма. В качестве критерия эффек- тивности сердечной ресинхронизирующей терапии указано не менее 93\% бивентрикулярных навязанных комплексов по данным опроса имплантированного устройства или ХМ ЭКГ. В данном случае хотелось бы обратить внимание на следующие дополнительные обстоятельства. Во-первых, в некоторых рекомендациях предусмотрен еще более жесткий критерий эффективности: более 98\% [7]. Во-вторых, частая ЖЭ, конечно, является не единственной причиной снижения процента навязанного бивентрикулярного ритма. В-третьих, ХМ ЭКГ, вероятно, имеет преимущество перед опросом имплантированного устройства, т.к., в отличие от последнего, позволяет выявить сливные и псевдосливные QRS-комплексы.

4. Оценку эффективности и безопасности подавления желудочковой эктопической активности при помощи ААП у больных со структурной патологией сердца / дисфункцией ЛЖ рекомендовано проводить под контролем повторного ХМ ЭКГ (УУР В, УДД 3 ЕОК, IIaC).

В следующем далее комментарии приведены критерии эффективности ААТ. Она может быть признана успешной при снижении общего количества ЖЭ более чем на $50 \%$ и/или уменьшении количества часов в сутки, в течение которых регистрировалась ЖЭ, более чем в 2 раза в сравнении с исходным (до лечения). Одновременно должно регистрироваться уменьшение количества парных ЖЭ в 10 раз и более, а также полное устранение неустойчивой желудочковой тахикардии.

Нельзя не отметить, что совсем немного внимания в рекомендациях уделено т.н. дополнительным электрокардиографическим методам исследования, реализованным в системах ХМ ЭКГ, таким как вариабельность сердечного ритма, микроальтернация Т-зубца, сигнал-усредненная ЭКГ, турбулентность сердечного ритма, вариабельность и динамика QT-интервала. Лишь дважды в тезис-рекомендациях упоминаются эти методы, причем один раз - с отрицательной коннотацией.

1. Сигнал-усредненная ЭКГ рекомендована для улучшения диагностики аритмогенной кардиомиопатии правого желудочка у пациентов с диагностированными желудочковыми нарушениями ритма или имеющих высокий риск развития жсизнеугрожающих желудочковых аритмий (УУР В, УДД 2, ЕОК ІВ).

2. Неинвазивные методы (например, оценка альтернации зубца T, дисфункции вегетативной системы или сигнал-усредненной ЭКГ) не рекомендуются для стратификации риска в ранний период после инфаркта миокарда (УУР С, УДД 4, ЕОК ІІІВ).

\section{БРАДИАРИТМИИ И НАРУШЕНИЯ ПРОВОДИМОСТИ}

Раздел рекомендаций «Диагностика» не содержит конкретных тезис-рекомендаций, имеющих непосредственное отношение к ХМ ЭКГ. Однако целесообразность его использования, вытекает, например, из такого положения.

Рекомендуется в процессе обследования проводить выявление и диагностику сопутствующих 
нарушений ритма сердца и аритмогенных электрокардиографических синдромов (УУР А, УДД 3, ЕОК - нет).

В подразделе «Электрокардиография» указано, что при постоянной форме брадиаритмий достаточно ЭКГ. Но при их интермиттирующем характере для выявления требуются методы длительного мониторирования ЭКГ.

Эти методы описаны в подразделе с соответствующим названием «Длительное мониторирование ЭКГ». В нем изложены представления о разных видах регистрации электрокардиосигнала. В их число, естественно, включены варианты ХМ ЭКГ от 24-48 часов до 7 суток. Кроме того, в подразделе перечислены амбулаторные устройства для интермиттирующей записи ЭКГ, а также непрерывные «регистраторы событий» с петлевой памятью, позволяющие фиксировать аритмические события на протяжении от 30 суток до 3 лет. Общие для длительного мониторирования ЭКГ тезис-рекомендации сформулированы следующим образом.

1. Для выявления брадикардии и нарушений проводимости, клинико-электрокардиографической корреляции с симптомами рекомендуется проведение длительного мониторирования сердечного ритма (УУР С, УДД 4, ЕОК - нет).

2. Рекомендуется выполнение длительного мониторинга сердечного ритма пациентам с документированной или подозреваемой брадикардией или нарушением проводимости для установления корреляции между частотой сердечных сокращений или нарущениями проводимости с симптомами с использованием кардиомонитора, выбранного на основе частоты, характера симптомов, а также предпочтения пациента (УУР С, УДД 4, ЕОК - нет).

3. Рекомендуется использовать различные виды длительного мониторирования ЭКГ в зависимости от частоты развития клинических симптомов, связанных с брадикардиями или нарушениями проводимости (УУР В, УДД 2, ЕОК - нет).

4. Рекомендуется при хронической брадиаритмии без гемодинамической нестабильности и при интермиттирующих редко возникающих брадиаритмиях проведение обследования пациента в амбулаторных условиях или в условиях госпитальной телеметрической регистрации ЭКГ (УУР В, УДД 2, EOK - нет).

В комментарии предложено небесспорное разделение на «норму» и «патологию» при дисфункции синусового узла и нарушениях АВ проводимости. Так, минимальной нормальной ЧСС в дневное время в покое предложено считать 40 в 1 мин, ночью - 35 в 1 мин (вне зависимости от пола и возраста). Максимальная синусовая пауза не должна превышать двух секунд. Допускаются, впрочем, отклонения от «нормы» у спортсменов высокой квалификации, лиц тяжелого физического труда, юношей. У них может выявляться более выраженная брадикардия, возможно, в сочетании с другими проявлениями дисфункции синусового узла. Однако нормальными эти показатели могут считаться только в отсутствие симптомов и при наличии адекватного прироста ЧСС во время физической нагрузки. Не следует считать патологией АВ блокаду I ст., причем не только транзиторную, но и постоянную (критерии ее функционального характера - узкие комплексы QRS, исчезновение блокады при физической нагрузке или пробе с атропином. Непатологической, не влияющей на прогноз предложено считать АВ блокаду II ст. 1-го типа (особенно ночью, во сне, у молодых здоровых лиц, спортсменов).

В подразделе приводится небольшая таблица, позволяющая выбрать оптимальный метод длительного мониторирования ЭКГ в зависимости от частоты развития эпизодов брадиаритмии (см. табл. 3). Из нее следует, что 7-суточное мониторирование не является для авторов рекомендаций одним из возможных вариантов ХМ ЭКГ. Принципиального значения это не имеет, приведем таблицу без изменений.

В целом важность ХМ ЭКГ для диагностики брадиаритмий и нарушений проводимости сердца подчеркивается тем, что в числе 10 положений раздела «Критерии оценки качества медицинской помощи» метод упомянут дважды. Вот эти положения.

Для выявления брадикардии и нарушений проводимости, клинико - электрокардиографической корреляции с симптомами рекомендуется проведение длительного мониторирования сердечного ритма.

1. Выполнен длительный мониторинг сердечного ритма у пациентов с документированной или подозреваемой брадикардией или нарущением проводимости для установления корреляции между частотой сердечных сокращений или нарушениями проводимости с симптомами с использованием кардиомонитора, выбранного на основе частоты, характера симптомов, а также предпочтения пациента.

2. Таким образом, при подозрении на наличие у пациента транзиторной брадиаритмии или наруиения проводимости должен быть использован ка-

Таблица 3.

Методы длительного мониторирования ЭКГ в зависимости от частоты развития эпизодов брадиаритмии

\begin{tabular}{|l|l|}
\hline Частота эпизодов & Метод и длительность мониторирования \\
\hline Ежедневно & $\begin{array}{l}\text { Суточное мониторирование 24 часа, госпитальное мониторирование или телеметрическая } \\
\text { регистрация ЭКГ }\end{array}$ \\
\hline Еженедельно и чаще & $\begin{array}{l}\text { Суточное мониторирование 24 часа, госпитальное мониторирование или телеметрическая } \\
\text { регистрация ЭКГ до 7 суток или наружный петлевой регистратор }\end{array}$ \\
\hline Ежемесячно & Наружный петлевой регистратор 14-30 суток \\
\hline Реже 1 раза в месяц & Имплантируемый петлевой регистратор \\
\hline
\end{tabular}


кой-либо из методов длительного мониторирования ЭКГ в соответствии с критериями, предложенными в табл. 3. Очевидно, что основным из этих.методов является именно ХМ ЭКГ.

\section{Рекомендации по проведению ХМ ЭКГ в соответствии с согласованным мнением экспертов HRS-ISHNE} 2017 года

\begin{tabular}{|c|c|c|}
\hline Рекомендации & КР & ДУ \\
\hline \multicolumn{3}{|l|}{ Выбор варианта ХМ ЭКГ } \\
\hline $\begin{array}{l}\text { ХМ ЭКГ в течение 24-48-часов рекомендовано при ожидаемой частоте симптомных событий, } \\
\text { соответствующей продолжительности мониторирования. }\end{array}$ & I & B-NR \\
\hline $\begin{array}{l}\text { Продолжительное ХМ ЭКГ (15-30 дней) рекомендовано при симптомных событиях с частотой } \\
\text { менее одного раза в день или же при неизвестной их частоте. }\end{array}$ & I & B-R \\
\hline $\begin{array}{l}\text { 12-канальное XM рекомендовано для анализа морфологии QRS-комплексов (ЖЭ, СРT), } \\
\text { изменений ST-сегмента (синдром Бругада, ишемия) и оценки QT-динамики. }\end{array}$ & I & $\mathrm{C}$ \\
\hline $\begin{array}{l}\text { Продолжительное ХМ (1-14 дней) показано для облегчения количественной оценки } \\
\text { и определения характера тяжести аритмии и ее динамики (например, ЖЭ, синусовая } \\
\text { тахикардия). }\end{array}$ & I & $\mathrm{B}-\mathrm{NR}$ \\
\hline \multicolumn{3}{|l|}{ Показания к ХМ ЭКГ } \\
\hline $\begin{array}{l}\text { ХМ ЭКГ рекомендовано при необъяснимых обмороках, если их возможной причиной м.б. } \\
\text { брадикардия или тахикардия, или имеется необходимость в их исключении. }\end{array}$ & I & B-R \\
\hline ХМ ЭКГ рекомендовано при необъяснимых сердцебиениях. & $\mathrm{I}$ & B-R \\
\hline $\begin{array}{l}\text { ХМ ЭКГ показано для оценки характера проводимости по аномальным путям и для } \\
\text { выявления эпизодов ФП у пациентов с синдромом WPW. }\end{array}$ & I & B-NR \\
\hline $\begin{array}{l}\text { ХМ ЭКГ показано пациентам с криптогенным инсультом для выявления недиагностированной } \\
\text { ранее ФП. }\end{array}$ & I & B-R \\
\hline $\begin{array}{l}\text { ХМ ЭКГ рекомендовано при недавно диагностированной неишемической кардиомиопатии, } \\
\text { если есть подозрение на обусловленную аритмией дисфункцию ЛЖ. }\end{array}$ & I & B-NR \\
\hline $\begin{array}{l}\text { ХМ ЭКГ м.б. полезным для выявления и количественной оценки ФП, а также связанных с } \\
\text { ней частотой желудочковых сокращений, триггерных аритмий (предсердной экстрасистолии, } \\
\text { НЖТ, ТП и брадикардии) и пауз ритма после кардиоверсии. }\end{array}$ & IIA & B-NR \\
\hline \multicolumn{3}{|l|}{ ХМ ЭКГ для оценки риска } \\
\hline $\begin{array}{l}\text { ХМ ЭКГ рекомендовано для выявления неустойчивой желудочковой тахикардии у пациентов } \\
\text { с гипертрофической кардиомиопатией для стратификации риска внезапной сердечной смерти. }\end{array}$ & I & B-NR \\
\hline $\begin{array}{l}\text { ХМ ЭКГ рекомендовано для количественной оценки ЖЭ и выявления неустойчивой } \\
\text { желудочковой тахикардии у пациентов с аритмогенной кардиомиопатией/дисплазией правого } \\
\text { желудочка для диагностики и стратификации риска. }\end{array}$ & I & B-NR \\
\hline $\begin{array}{l}\text { ХМ ЭКГ рекомендовано амбулаторным пациентам, начинающим лечение ААП, для } \\
\text { выявления их проаритмического эффекта. }\end{array}$ & I & $\mathrm{C}$ \\
\hline $\begin{array}{l}\text { ХМ ЭКГ м.б. полезным для оценки эффективности подавления аритмии с помощью } \\
\text { медикаментозного или интервенционного лечения. }\end{array}$ & IIA & B-NR \\
\hline $\begin{array}{l}\text { 24-48 - часовое ХМ ЭКГ м.б. полезным для пациентов с перенесенным инфарктом } \\
\text { миокарда и пограничной фракцией выброса ЛЖ в пределах 35\%-40\% для выявления } \\
\text { неустойчивой желудочковой тахикардии и последующей стратификации риска с помощью } \\
\text { электрофизиологического исследования с целью определения показаний для имплантации } \\
\text { кардиовертера-дефибриллятора. }\end{array}$ & IIA & B-R \\
\hline $\begin{array}{l}\text { ХМ ЭКГ м.б. полезным для пациентов с электрокардиостимулятором и симптомами, } \\
\text { возможно, связанными с дисфункцией имплантированного антиаритмического устройства, } \\
\text { если опрос устройства недостаточно информативен. }\end{array}$ & IIA & B-NR \\
\hline $\begin{array}{l}\text { Использование ХМ ЭКГ м.б. рассмотрено для оценки прогноза и стратификации риска у } \\
\text { пациентов с неишемической кардиомиопатией. }\end{array}$ & IIB & B-NR \\
\hline $\begin{array}{l}\text { Использование 24-48 - часового ХМ ЭКГ м.б. рассмотрено после острого инфаркта миокарда } \\
\text { для выявления неустойчивой желудочковой тахикардии с целью прогнозирования увеличения } \\
\text { риска внезапной сердечной смерти, особенно у пациентов со сниженной фракцией выброса Л久 }\end{array}$ & IIB & B-NR \\
\hline
\end{tabular}

В заключение можно констатировать, что метод ХМ ЭКГ является одним из основных при обследовании пациентов с нарушениями ритма и проводимости сердца. Ранее в своей публикации, посвященной ана-

Таблица 4.

Примечание. КР - класс рекомендаций, ДУ - доказательный уровень. 
лизу согласованного мнения экспертов HRS-ISHNE по амбулаторной ЭКГ и наружному мониторированию деятельности сердца / телеметрии мы писали, что одним из самых важных достоинств этого документа является то, что что «...он перекликается со всеми имеющимися современными рекомендациями по диагностике и лечению, как интервенционному, так и медикаментозному, нарушений ритма и проводимости сердца, учитывает написанное в них и практически ни в чем не противоречит» [8]. Попробуем теперь, напротив, взглянуть, насколько полно возможности ХМ ЭКГ, рассмотренные в согласованном мнении экспертов, использованы в наших национальных рекомендациях по аритмиям. Помочь в этом сравнении нам могут выводы комитета экспертов, представленные в виде таблицы, составленной из рекомендаций с привычными нам классами (I, IIA, IIB и III) и непривычными доказательными уровнями (A, B-R, B-NR, E и C). Доказательный уровень A - самый высокий, т.к. доказательства получены в нескольких клинических исследованиях с реестрами или без них. Доказательный уровень В - умеренный, полученный либо в рандомизированных исследованиях (BR), либо в хорошо выполненных нерандомизированных исследованиях (B-NR). Доказательный уровень C основан на данных слабых исследований со значительными ограничениями (например, рандомизированные или нерандомизированные наблюдательные исследования или реестры; исследования с ограничениями дизайна или исполнения; мета-анализы таких исследований; физиологические или механистические исследования у людей). Наконец, доказательный уровень Е является просто консенсусом мнений экспертов на основании клинического опыта, когда доказательства недостаточны, туманны или противоречивы. Согласитесь, это очень похоже на структуру УДД российских рекомендаций. Итак, рассмотрим табл. 4.
Из сравнения с этой таблицей следует, что использование не только суточного, но и многосуточного ХМ ЭКГ обсуждается в российских рекомендациях достаточно подробно. При этом целесообразность 12-канального ХМ ЭКГ для анализа контура ЭКГ (например, при предполагаемом синдроме Бругада), оценки морфологии QRS-комплексов при желудочковых аритмиях, к сожалению, не оговаривается. Между тем, достаточно давно обсуждаемая возможность ориентировочной оценки топики ЖЭ по 12-ти отведениям ЭКГ $[9,10]$ с высокой долей вероятности будет реализована в холтеровских системах уже в ближайшее время. Впрочем, и без того 12-канальная запись становится «золотым стандартом» ХМ ЭКГ. Необходимость в ХМ ЭКГ при обследовании пациентов с сердцебиением, брадикардией, синкопальными состояниями, синдромом (феноменом) WPW, криптогенными инсультами, подозрением на тахикардиомиопатию рассмотрена в достаточно полной мере. В меньшей степени отражены возможности метода для оценки «бремени» ФП, триггерных аритмий. Весьма подробно описано применение ХМ ЭКГ для контроля эффективности и безопасности ААТ. В то же время не обсуждается подробно использование метода для стратификации риска внезапной сердечной смерти в конкретных клинических ситуациях: при гипертрофической кардиомиопатии, аритмогенной кардиомиопатии правого желудочка, дилатационной кардиомиопатии, а также у пациентов, перенесших инфаркт миокарда, особенно с низкой фракцией ЛЖ. К сожалению, в рекомендациях по брадиаритмиям и желудочковым аритмиям не отражены возможности ХМ ЭКГ для наблюдения за пациентами с электрокардиостимуляторами и другими имплантируемыми антиаритмическими устройствами, за исключением сердечной ресинхронизирующей терапии.

\section{ЛИТЕРАТУРА}

1. Омельяновский ВВ, Авксентьева МВ, Железнякова ИА и др. Клинические рекомендации как инструмент повышения качества медицинской помощи. Онкопедиатрия. 2017;4(4): 247-258. [Omeljanovskij VV, Avksent'eva MV, Zhelezniakova IA, et al. Clinical Guidelines as a Tool for Improving the Quality of Medical Care Delivery. Onkopediatria. 2017;4(4): 247-258. (In Russ.)]. 2. Омельяновский BВ, Сухоруких ОА, Лукьянцева ДВ и др. Методические рекомендации по проведению оценки научной обоснованности включаемой в клинические рекомендации информации. ЦЭККМП, 2019 г. Доступно: https://rosmedex.ru/wp-content/ uploads/2019/10/mr_nauch-obosn-kr.pdf. [Omeljanovskij VV, Sukhorukikh OA, Lukyantseva DV et al. Guidelines for assessing the scientific validity of information included in clinical guidelines. Center for Healthcare Quality Assessment and Control, 2019. Available from: https://rosmedex.ru/wp-content/uploads/2019/10/mr_nauch-obosn-kr. pdf (In Russ.)]

3. Kirchhof P, Benussi S, Kotecha D, et al. 2016 ESC Guidelines for the management of atrial fibrillation developed in collaboration with EACTS. Eur Heart J. 2016;37(38): 2893-2962. DOI:10.1093/eurheartj/ehw210.
4. Hindricks G, Potpara T, Dagres N, et al. 2020 ESC Guidelines for the diagnosis and management of atrial fibrillation developed in collaboration with EACTS. Eur Heart J. 2020 Aug 29; ehaa612. DOI: 10.1093/eurheartj/ ehaa612.

5. Тихоненко ВМ, Тулинцева ТЭ, Лышова ОВ и др. Нарушения ритма и проводимости сердца у здоровых лиц. Вестник аритмологии. 2018;91: 11-21. [Tikhonenko VM, Tulintseva TE, Lyshova OV et al. Cardiac arrhythmias in healthy subjects. Journal of Arrhythmology. 2018;(91):11-20. (In Russ.)]

6. Pedersen CT, Kay GN, Kalman J, et al. EHRA/HRS/ APHRS expert consensus on ventricular arrhythmias. Europace. 2014; 16: 1257-1283.

7. Steinberg JS, Varma N, Cygankiewicz I, et al. 2017 ISHNE-HRS expert consensus statement on ambulatory ECG and external cardiac monitoring / telemetry. Heart Rhythm. 2017;14(7): e55-e96.

8. Шубик ЮВ, Батурова МА, Трегубов АВ. Рекомендации по холтеровскому мониторированию электрокардиограммы: прошлое, настоящее, будущее. Вестник аритмологии. 2018;94: 57-67 [Shubik YuV, Baturova MA, Tregubov AV. Guidance on ECG Holter monitoring: past, 
present, future. Journal of Arrhythmology. 2018;(94):5767. (In Russ.)] DOI: 10.25760/VA-2018-94-57-67.

9. Вайнштейн АБ, Яшин СМ, Думпис ЯЮ, Шубик ЮВ. Электрокардиографическая топическая диагностика некоронарогенных правожелудочковых аритмий. Вестник аритмологии. 2004;(34): 11-17. [ECG diagnostics of non-coronary right ventricular arrhythmias. Journal of Arrhythmology. 2004;(34): 11-17. (In Russ.)].
10. Ревишвили АШ, Носкова МВ, Рзаев ФГ, Артюхина EA. Неинвазивная топическая диагностика некоронарогенных желудочковых аритмий. Вестник аритмологии. 2004;(35): 5-15. [Revishvili Ash, Noskova MV, Rzaev FG, Artyukhina YeA. Non-invasive diagnostics of non-coronary ventricular arrhythmias. Journal of Arrhythmology. 2004;(35): 5-15]. 